\title{
Mitochondrial Function could be one Novel Therapeutic Target to in Effects of Exercise to Prevent Post-Traumatic Stress Disorder
}

\author{
Lina Sun*, Kai Cui and Rundong Liang \\ College of PE and Sport, Beijing Normal University, Beijing, China
}

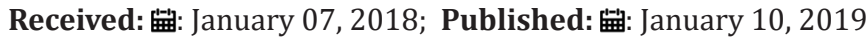

*Corresponding author: Lina Sun, College of PE and Sport, Beijing Normal University, Beijing, China

\begin{abstract}
Post-traumatic stress disorder (PTSD) is a serious mental disorder to threaten the life and career of the patients, which highly associated with the neural plasticity at hippocampus. Although previous studies reveal multiple factors acting as the biological bases involving with the development of PTSD, mitochondrial functions could be a new direction for investigating the pathology and therapy of the disease. Recent study suggested improvement of mitochondrial functions is one of the major advantage of physical exercise, which has been widely recognized as the helpful life style in preventing not only psychiatric disorders but also numbers of complex diseases including neurodegeneration, diabetes or even some types of cancer. Thus, mitochondrial functions would be the new target in preventing PTSD or promoting its recovery during treatments. In this minireview, we summarized the relationship between mitochondrial functions and psychiatric disorder like PTSD and depression. Moreover, based on the benefits of exercise in improving the brain functions via affecting mitochondrial biological status, we proposed that mitochondrial functions are the key to regulate neural functions and to prevent the exacerbation of PTSD.
\end{abstract}

Keywords: PTSD; Mitochondria; Exercise; Neural plasticity; Hippocampus

\section{Mini Review}

Post-traumatic stress disorder (PTSD) is a mental health condition that is triggered by panic memory. It could induce a serious of psychiatric symptoms including avoidance, negative thinking and mood as well as changes in physical and emotional reactions [1,2]. From the angle of pathophysiology, impaired neural plasticity contributes the development of PTSD especially and resulted in the abnormal sensitivity of the patients to certain environmental cues $[3,4]$. Therefore, how to maintain or reserve neural plasticity and neural symptoms is urgent for solution to develop the effective treatment to PTSD.

Improvement of the neural plasticity especially the adult neurogenesis and synaptic functions at hippocampus is critical to maintain the adaptability of the brain under psychiatric stressors. In adult hippocampus, neural plasticity including neurogenesis and synaptic plasticity are mediated by multiple factors. They serve as the key physiological functions needed for recovered in treating psychiatric disorders like depression, PTSD and bipolar disorder [5-7]. Adult neurogenesis, composed by the self-renewal and fate commitment of neural stem cells (NSCs) as well as the maturation of neural progenitor cells (NPCs), offer the regenerative resource of the neuron population that helps to clear the panic memory $[4,8]$. Normalized neurogenesis at hippocampus was shown to promote the pattern separation behavior, which enhance the animal to control the emotion in the represented panic scenario [9]. Such capacity is the key for recovery from PTSD. Impairment of neurogenesis results in overgeneralization of fear, which subsequently manifests as inappropriate, uncontrollable expression of fear in neutral and safe environments [10]. Apart from neurogenesis, synaptic plasticity including the synaptogenesis like dendritic spine formation as well as the synaptic functions like long-term potentiation (LTP) and presynaptic plasticity also associating with development of depression.

Immobilization-stressed mice presented intensified fear memory and enhanced long-term potentiation (LTP) [11]. Deficiency of brain-derived neurotrophic factor (BDNF) and its intracellular kinase-activating receptor TrkB, have been implicated in the neurobiological mechanisms underlying the clinical manifestations of PTSD [12]. By offering different renewed resource such as at 
neuronal population as well as synaptic complexity, neural plasticity including neurogenesis and synaptic plasticity support the capacity of the brain to cope with the psychiatric stressors (Figure 1). Conversely, impairment of the neural plasticity is often showing to correlation with the occurrence of psychiatric disorder especially
PTSD or major depression [13,14]. Although different efforts were tried to improve the neural plasticity, inefficiency treatment effects or relapse of the disease symptom usually happen due to lack of understanding on the key regulator of the neural plasticity.

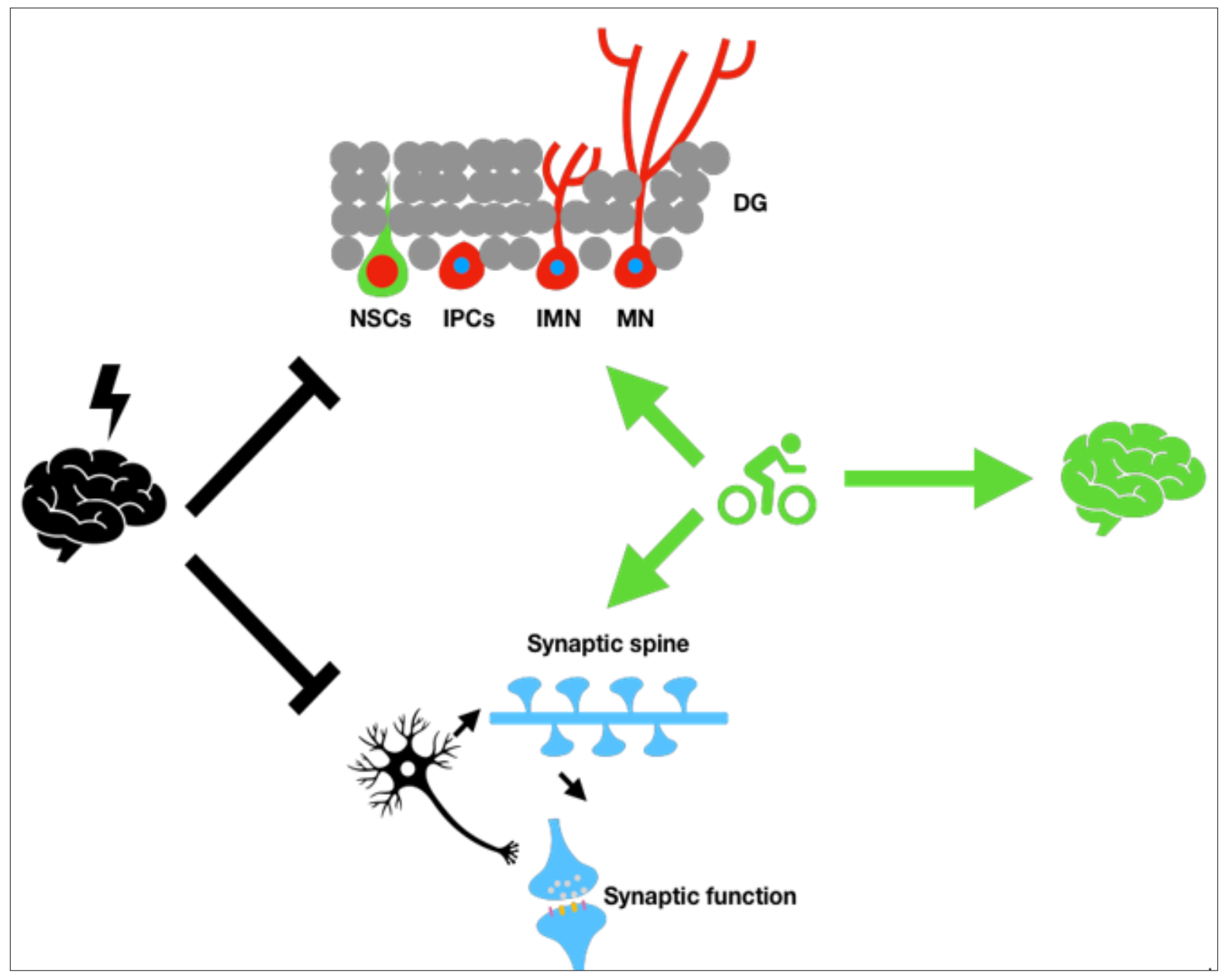

Figure 1: Exercise promote the recovery of patients with PTSD by promoting neural plasticity.

Hippocampus is the limbic system in brain with the high structural and functional plasticity. Panic memories due to its strong negative feelings usually form serious psychiatric stressor, which impairs the neural plasticity in hippocampus including neurogenesis and synaptic functions. Neurogenesis mainly including the process of self-renewal of the neural stem cells (NSCs), development of intermediate progenitor cells (IPCs), the maturation of the immature neurons (IMN) as well as the survival of new generated mature neurons (MN). Synaptic plasticity including the density of the post synaptic spines, as well as the functions of single synaptic that containing the functions of pre- and post- synapse. It has been widely reported that exercise could promote those two kinds of neural plasticity and improve the hippocampal functions. Such mechanism explains the effects of exercise in attenuating the main symptoms of PTSD.

The effects of physical exercise, the effective and economic way to prevent and help the recovery the psychiatric symptom associated with PTSD, enlighten the new idea that metabolic functions could be a key target in regulating neural plasticity [1517]. Convergent of evidence indicates the role of mitochondria in regulating neural functions. At hippocampus, adult NSCs undergo the switch from glycolysis to oxidative phosphorylation (OXPHOS) following the process of neurogenesis [18]. In mature neurons, mitochondrial metabolism is the main energy source enabling the stimulation of complex electrophysiological process $[19,20]$. Coincidentally, physical exercise is the effective way to promote mitochondrial functions and perform the therapeutic effects in many chronic diseases, like diabetes, neurodegeneration and psychiatric disorders $[21,22]$. Additionally, drugs that widely used 
on clinic was proved could improve the mitochondria functions and thus perform the brain protection.

For example, brain functional recovery drug piracetam was proved could prevent ageing induced neurogenesis decline by promote mitochondrial metabolism [18]. Antioxidants that targeted on controlling the level of reactive oxidative species (ROS) could also reserve the functions of mitochondria [23]. However, it is noteworthy that exercise may elevate the level of ROS, which has been recently declared as the mechanism in antidiabetic effects [24]. Consistent with such point, certain level of ROS serves as the 'second messenger' to promote the fate commitment of the NSCs at physiological level [25]. Based on such fact, exercise maybe the effective way to control the level of ROS into physiological reasonable by promoting mitochondrial OXPHOS. For synaptic functions, it was demonstrated that ablation of mitochondrial component uncouple protein 2 (UCP2) induced an anxiety prone, cognitively impaired behavioral phenotype, indicating the key roles of mitochondria functions in regulating synaptic plasticity [26]. Thus, normalization of mitochondrial functions could be the new direction on treatment of PTSD.

\section{Summary and Perspective}

It is well-recognized about the benefit of exercise to improve neural functions. However, something that showing beneficial may also lead a harmful result. Likewise, whether overproduction of ROS induced by inappropriate exercise will exacerbate the PTSD associated symptoms remain unknown. With deepen understanding on the relationship between exercise with neural plasticity during the recovery from PTSD, answering the question if certain level of exercise is helpful to improve mitochondrial functions or excessive intensity of exercise can result the adverse effects is worthwhile for explaining. Moreover, how to combine the personality exercise scheme with current clinical medications in different types of PTSD is also need for further investigation. In future research works, focusing on improving mitochondrial functions to promote neuroprotective effects could the breakthrough to treat psychiatric disorders like PTSD and depression.

\section{References}

1. Cohen JA, Deblinger E, Mannarino AP, Steer RA (2004) A multisite, randomized controlled trial for children with sexual abuse-related PTSD symptoms. J Am Acad Child Adolesc Psychiatry 43(4): 393-402.

2. Raskind MA, Peskind ER, Kanter ED, Petrie EC, Radant A, et al. (2003) Reduction of nightmares and other PTSD symptoms in combat veterans by prazosin: A placebo-controlled study. Am J Psychiatry 160(2): 371373.

3. Adamec R, Kent P, Anisman H, Shallow T, Merali Z (1998) Neural plasticity, neuropeptides and anxiety in animals--implications for understanding and treating affective disorder following traumatic stress in humans. Neurosci Biobehav Rev 23(2): 301-318.

4. Kheirbek MA, Klemenhagen KC, Sahay A, Hen R (2012) Neurogenesis and generalization: A new approach to stratify and treat anxiety disorders. Nature neuroscience 15(12): 1613-1620.

5. Adamec RE, Blundell J, Burton P (2006) Relationship of the predatory attack experience to neural plasticity, pCREB expression and neuroendocrine response. Neurosci Biobehav Rev 30(3): 356-375.
6. Nissen C, Holz J, Blechert J, Feige B, Riemann D, et al. (2010) Learning as a model for neural plasticity in major depression. Biol Psychiatry 68(6): 544-552.

7. Phillips ML, Ladouceur CD, Drevets WC (2008) A neural model of voluntary and automatic emotion regulation: implications for understanding the pathophysiology and neurodevelopment of bipolar disorder. Mol Psychiatry 13(9): 833-857.

8. Clelland CD, Choi M, Romberg C, Clemenson GD, Fragniere A, et al. (2009) A functional role for adult hippocampal neurogenesis in spatial pattern separation. Science 325(5937): 210-213.

9. Sahay A, Scobie KN, Hill AS, O'Carroll CM, Kheirbek MA, et al. (2011) Increasing adult hippocampal neurogenesis is sufficient to improve pattern separation. Nature 472(7344): 466-470.

10. Besnard A, Sahay A (2016) Adult Hippocampal Neurogenesis, Fear Generalization and Stress. Neuropsychopharmacology 41(1): 24-44.

11. Nijholt I, Farchi N, Kye M, Sklan EH, Shoham S, et al. (2004) Stressinduced alternative splicing of acetylcholinesterase results in enhanced fear memory and long-term potentiation. Mol Psychiatry 9(2): 174-183.

12. Kozlovsky N, Matar MA, Kaplan Z, Kotler M, Zohar J, et al. (2007) Long-term down-regulation of BDNF mRNA in rat hippocampal CA1 subregion correlates with PTSD-like behavioural stress response. The international journal of neuropsychopharmacology / official scientific journal of the Collegium Internationale Neuropsychopharmacologicum 10(6): 741-758.

13. Kunas SL, Yang Y, Straube B, Kircher T, Gerlach AL, et al. (2018) The impact of depressive comorbidity on neural plasticity following cognitive- behavioral therapy in panic disorder with agoraphobia. J Affect Disord 245: 451-460.

14. Olson DE (2018) Psychoplastogens: A Promising Class of PlasticityPromoting Neurotherapeutics. J Exp Neurosci 12: 1179069518800508.

15. Rosenbaum S, Sherrington C, Tiedemann A (2015) Exercise augmentation compared with usual care for post-traumatic stress disorder: A randomized controlled trial. Acta Psychiatr Scand 131(5): 350-359.

16. Shivakumar G, Anderson EH, Suris AM, North CS (2017) Exercise for PTSD in Women Veterans: A Proof-of-Concept Study. Mil Med 182(11): e1809-e1814.

17. Whitworth JW, Ciccolo JT (2016) Exercise and Post-Traumatic Stress Disorder in Military Veterans: A Systematic Review. Mil Med 181(9): 953-960.

18. Beckervordersandforth R, Ebert B, Schaffner I, Moss J, Fiebig C, et al. (2017) Role of Mitochondrial Metabolism in the Control of Early Lineage Progression and Aging Phenotypes in Adult Hippocampal Neurogenesis. Neuron 93(3): 560-573.

19. Kann O (2016) The interneuron energy hypothesis: Implications for brain disease. Neurobiology of disease 90: 75-85.

20. Kwon SK, Sando R 3rd, Lewis TL, Hirabayashi Y, Maximov A, et al. (2016) LKB1 Regulates Mitochondria-Dependent Presynaptic Calcium Clearance and Neurotransmitter Release Properties at Excitatory Synapses along Cortical Axons. PLoS Biol 14: e1002516.

21. Barnes JN (2015) Exercise, cognitive function and aging. Adv Physiol Educ 39(2): 55-62.

22. Jenkins DW, Jenks A (2017) Exercise and Diabetes: A Narrative Review. J Foot Ankle Surg 56(5): 968-974.

23. Oyewole AO, Birch-Machin MA (2015) Mitochondria-targeted antioxidants. FASEB journal: official publication of the Federation of American Societies for Experimental Biology 29(12): 4766-4771.

24. Watson JD (2014) Type 2 diabetes as a redox disease. Lancet 383(9919): 841-843. 
25. Khacho M, Clark A, Svoboda DS, Azzi J, MacLaurin JG, et al. (2016) Mitochondrial Dynamics Impacts Stem Cell Identity and Fate Decisions by Regulating a Nuclear Transcriptional Program. Cell stem cell 19(2): 232-247.

\section{ISSN: 2574-1241}

DOI: $10.26717 / B J S T R .2019 .13 .002340$

Lina Sun. Biomed J Sci \& Tech Res

This work is licensed under Creative

Commons Attribution 4.0 License

Submission Link: https://biomedres.us/submit-manuscript.php
26. Hermes G, Nagy D, Waterson M, Zsarnovszky A, Varela L, et al. (2016) Role of mitochondrial uncoupling protein-2 (UCP2) in higher brain functions, neuronal plasticity and network oscillation. Mol Metab 5(6): 415-421.

$\begin{array}{ll}\text { BIOMEDICAL } & \text { Assets of Publishing with us } \\ \text { RESEARCHES } & \text { - Global archiving of articles } \\ & \text { - Immediate, unrestricted online access } \\ & \text { - Rigorous Peer Review Process } \\ & \text { - Authors Retain Copyrights }\end{array}$

